\title{
ANÁLISE FATORIAL CONFIRMATÓRIA DA DEPRESSION ANXIETY STRESS SCALE EM PESSOAS COM DOENÇA RENAL CRÓNICA
}

\author{
| Luís Sousa'; Cristina Marques-Vieira²; Sandy Severino³; José Carlos Gomes; Helena Maria Guerreiro José5 |
}

\section{RESUMO}

CONTEXTO: A Depression Anxiety Stress Scale (DASS) foi desenvolvida por Lovibond e Lovibond, em 1995, para avaliar os sintomas da ansiedade e da depressão. A versão portuguesa da DASS com 21 itens (DASS-21) confirmou ter propriedades idênticas às da versão original.

OBJETIVO: Confirmar a estrutura da DASS-21 em pessoas com doença renal crónica em programa de hemodiálise.

MÉTODO: Estudo metodológico. Foi obtida uma amostra representativa de 159 pessoas com doença renal cónica, em programa de hemodiálise, em duas clínicas e num serviço de nefrologia de um Centro Hospitalar, todos em Lisboa, Portugal. Foi realizada colheita de dados de maio a junho de 2015. Desenvolveu-se uma análise fatorial confirmatória com recurso ao software AMOS. Utilizaramse os seguintes parâmetros: rácio entre o Qui quadrado e os graus de liberdade (X2/g.l), goodness-of-fit index (GFI), comparative fit index (CFI), Tucker-Lewis index (TLI) e root mean square error of approximation (RMSEA).

RESULTADOS: Obteve-se X2/g.l=1,980, GFI $=0,84, \mathrm{CFI}=0,87$, TLI $=0,85$ e RMSEA $=0,08$, o que demostra ser um bom ajustamento para a hipótese da solução de dois fatores. Não se confirma a solução proposta na versão original e na versão portuguesa (três fatores). CONCLUSÕES: A versão portuguesa da DASS-21 apresenta dois fatores, e é válida para medir stresse/ansiedade e depressão, em pessoas com doença renal crónica.

\section{PALAVRAS-CHAVE: Insuficiência Renal Crónica; Depressão; Ansiedade; Estudos de validação.}

\section{RESUMEN}

"Análisis factorial confirmatório da Depression Anxiety Stress Scale en enfermedad renal crónica"

CONTEXTO: La Escala de Depresión Ansiedad Estrés (DASS) fue desarrollada por Lovibond y Lovibond en 1995 con el objetivo de evaluar los síntomas de ansiedad y depresión. La versión en portugués de la DASS con 21 artículos (DASS-21) confirmó que tiene propiedades similares a la versión original.

OBJETIVO: Confirmar la estructura de la DASS-21 en las personas con enfermedad renal crónica en hemodiálisis.

MÉTODO: Estudio metodológico. Se obtuvo una muestra representativa de 159 personas con enfermedad renal crónica sometidos a hemodiálisis en dos clínicas en la ciudad de Lisboa y en el servicio de nefrología de un hospital también en Lisboa, Portugal. La cosecha se realizó entre mayo y junio de 2015. Se desarrolló un análisis factorial confirmatorio mediante el software AMOS. Los parámetros utilizados fueron: relación de la chicuadrado y los grados de libertad (X2 / gl) goodness-of-fit index (GFI), comparative fit index (CFI), Tucker-Lewis index (TLI) y lo root mean square error of approximation (RMSEA).

RESULTADOS: Se obtuvieron X2 / $\mathrm{df}=1,980$, GFI $=0,84$, CFI $=0,87$, TLI $=0,85$ y RMSEA $=0,08$, lo que demuestra ser un buen ajuste a la hipótesis de la solución de dos factores. No se ha confirmado la solución propuesta en la versión original ni en la versión en Inglés (tres factores). CONCLUSIONES: La versión portuguesa de la DASS 21 tiene dos factores, y es válido para medir el estrés / ansiedad y la depresión en personas con enfermedad renal crónica.

DESCRIPTORES: Insuficiencia Renal Crónica; Depresión; Ansiedad; Estudios de validación.

Submetido em 22-11-2016

Aceite em 20-02-2017

\section{ABSTRACT \\ "Confirmatory Factor Analysis of the Depression Anxiety Stress Scale in Chronic Kidney Disease"}

BACKGROUND: The Depression Anxiety Stress Scale (DASS) was developed by Lovibond \& Lovibond in 1995 with the objective of evaluating the symptoms of anxiety and depression. The Portuguese version of the DASS with 21 items (DASS-21) confirmed having identical properties as those of the original version.

AIM: Confirm the structure of DASS-21 in people with chronic kidney disease on dialysis.

METHOD: Methodological study. A representative sample of 159 people with chronic kidney disease on dialysis was obtained in two clinics in the Lisbon region and a nephrology department in a Lisbon hospital center, Portugal. Data collection occurred from may to june 2015. A confirmatory factor analysis was developed using the AMOS software. The following parameters were used: ratio between Chi squared and the degrees of freedom (X2/g.l), goodness-of-fit index (GFI), comparative fit index (CFI), Tucker-Lewis index (TLI) and root mean square error of approximation (RMSEA).

RESULTS: The following was obtained:X2/g.l $=1,980$, GFI $=0,84, \mathrm{CFI}=0,87$, TLI $=0,85$ and RMSEA $=0,08$, which shows being a good adjustment for the hypothesis of the solution of two factors. The solution proposed in the original version and in the Portuguese version is not confirmed (three factors).

CONCLUSIONS: The portuguese version of the DASS presents two factors and is valid for measuring Stress/Anxiety and Depression, in people with chronic kidney disease.

KEYWORDS: Chronic Renal Insufficiency; Depression; Anxiety; Validation studies.

1 Mestre; Enfermeiro Especialista em Enfermagem de Reabilitação; Enfermeiro no Centro Hospitalar Lisboa Central, Lisboa e Professor Adjunto na Universidade Atlântica, Fábrica da Pólvora de Barcarena, 2730-036 Barcarena, Oeiras, Portugal, luismmsousa@gmail.com

2 Mestre; Enfermeira Especialista em Enfermagem de Reabilitação; Professora na Universidade Católica Portuguesa, cristina_marques@ics.lisboa.ucp.pt

3 Mestre; Enfermeira Especialista em Enfermagem de Reabilitação; Enfermeira no Centro Hospitalar Lisboa Central, Lisboa, sandyseverino@gmail.com

4 Doutor em Saúde Pública. Especialista em Enfermagem de Saúde Mental e Psiquiatria. Professor Coordenador na Escola Superior de Saúde do Instituto Politécnico de

Leiria. Portugal, jcrgomes@ipleiria.pt

5 Doutor em Enfermagem. Especialista em Enfermagem Médico-cirúrgica. Diretora da Escola de Saúde Multiperfil, Luanda, Angola, helena.jose@multiperfil.co.ao 


\section{INTRODUÇÃO}

$\mathrm{O}$ aumento da prevalência da doença renal crónica (DRC) tem trazido desafios para a saúde pública, sendo esta considerada um problema social e económico em todo o mundo. Está associada a inúmeras comorbidades e a elevados gastos no âmbito da saúde (Fassbinder, Winkelmann, Schneider, Wendland \& Oliveira, 2015; Pinho, Silva \& Pierin, 2015). A DRC é definida como uma condição progressiva e irreversível das funções endócrinas, tubular e glomerular dos rins e tem como característica principal a diminuição do filtrado glomerular durante um período superior a três meses (Santos, Oliveira, Nunes, Barbosa \& Gouveia, 2015). A hemodiálise é o tratamento mais adotado na substituição da função renal. Esta é caracterizada por um processo mecânico e extracorpóreo, que consiste na extração de substâncias tóxicas e do excesso de líquido do organismo, sendo realizado três vezes por semana, com duração média de quatro horas (Costa \& Coutinho, 2016).A DRC introduz diversas alterações na vida da pessoa com repercussões a nível físico, psicológico, familiar e social (Ramos et al., 2015).

A doença e o stresse gerados pelo tratamento podem desencadear na pessoa com DRC medo, insegurança, ansiedade, depressão, baixa autoestima e sensação de inutilidade (Cavalcante, Lamy, Santos \& Costa, 2015). A depressão é das situações mais prevalentes nas pessoas com DRC e mediante o instrumento aplicado, os níveis de depressão podem atingir os 100\% (Costa \& Coutinho, 2016).

A Depression Anxiety Stress Scale (DASS) foi construída por Lovibond e Lovibond (1995) para avaliar sintomas de ansiedade e depressão, contudo, no estudo de validação emergiu uma dimensão relacionada com o stresse. Esta incluía os itens sobre a dificuldade em relaxar, tensão nervosa, irritabilidade e agitação. As versões portuguesas da DASS com 21 itens (DASS21), em pessoas saudáveis, confirmaram o modelo tripartido: depressão, ansiedade e stresse (Ribeiro, Honrado \& Leal, 2004; Pinto, Martins, Pinheiro \& Oliveira, 2015), assim como, as versões dos Estados Unidos da América (Sinclair et al., 2012), da Indonésia, de Singapura, do Sri Lanka e Tailândia (Oei, Sawang, Goh \& Mukhtar, 2013), do Vietname (Tran, Tran \& Fisher, 2013), do Brasil (Vignola \& Tucci, 2014) e da Austrália (Gomez, Summers, Summers, Wolf \& Summers, 2014). Contudo a versão italiana sugere um modelo de distresse geral com três dimensões ortogonais (Bottesi et al., 2015).
Em estudos realizados em Portugal, em pessoas com doença mental ( $\mathrm{n}=101)$, a DASS-21 revelou uma estrutura bifatorial (Apóstolo, Mendes \& Azevedo, 2006), porém, numa amostra recolhida em centros de saúde $(\mathrm{n}=1.301)$ confirmou o modelo tripartido (Apóstolo, Figueiredo, Mendes \& Rodrigues, 2011). Nesta investigação pretende-se responder à seguinte questão: será que o modelo de três fatores da DASS-21 (Lovibond \& Lovibond, 1995), em pessoas com DRC, é o que se ajusta melhor aos dados? De onde emerge o seguinte objetivo: confirmar a estrutura da DASS21 em pessoas com doença renal crónica (DRC) em programa de hemodiálise.

\section{METODOLOGIA}

Estudo metodológico (Lima, 2011), realizado, entre março e junho de 2015, num serviço de Nefrologia de um Centro Hospitalar e em duas unidades de diálise da Clínica Diaverum, ambos na região de Lisboa. A população do estudo foi constituída por pessoas com DRC em programa de hemodiálise. Como critérios de inclusão estabeleceu-se: pessoas com DRC, sujeitas a tratamento hemodialítico há pelo menos seis meses, com idade igual ou superior a 18 anos. Foram excluídas as pessoas com défice cognitivo e doença psiquiátrica ativa. Os dados sobre os critérios de exclusão foram colhidos no processo clínico e validados com o médico assistente de cada pessoa. Foi feita seleção aleatória da amostra. Para a colheita de dados foram utilizados: versão portuguesa da DASS-21 para medir o stresse, ansiedade e depressão (Ribeiro, Honrado \& Leal, 2004), e um instrumento para caracterizar o perfil da amostra, tanto ao nível sociodemográfico, como clínico (idade, género, nacionalidade, escolaridade, atividade profissional, estado civil, tempo de diálise, diagnóstico médico de hipertensão arterial e de diabetes).

A DASS-21 é constituída por 21 itens que se organizam em três subescalas, depressão, ansiedade e stresse, cada uma constituída por sete itens. Cada escala inclui vários conceitos, designadamente: depressão e disforia (um item); desânimo, (um item); desvalorização da vida (um item); autodepreciação (um item); falta de interesse ou de envolvimento (um item); anedonia (um item); inércia (um item); ansiedade e excitação do sistema autónomo (três itens); efeitos músculo-esqueléticos (um item); ansiedade situacional (um item); experiências subjetivas de ansiedade (dois itens); stresse -dificuldade em relaxar 
(dois itens); excitação nervosa (um item); facilmente agitado/chateado (um item); irritável/reação exagerada (dois itens); e impaciências (um item). As três escalas são compostas por sete itens cada, no total de 21 itens. Cada item consiste numa afirmação, que remete para sintomas emocionais negativos. Pede-se ao respondente que verifique a afirmação que melhor se aplica ao que viveu na semana anterior. Para cada frase existem quatro possibilidades de resposta, apresentadas numa escala tipo Likert. Os respondentes avaliam a extensão em que experimentaram cada sintoma durante a última semana, numa escala de quatro pontos de gravidade ou frequência: "não se aplicou nada a mim" até "aplicou-se a maior parte das vezes”. Os resultados de cada subescala são determinados pela soma dos resultados dos sete itens. A escala fornece três classificações, uma por cada subescala, numa variação de zero a 21. As notas mais elevadas em cada escala correspondem a estados afetivos mais negativos. A consistência interna foi examinada com recurso ao Alfa de Cronbach e os resultados encontrados para a EADS foram respetivamente de 0,85 para a escala de depressão, de 0,74 para a de ansiedade e de 0,81 para a de stresse (Ribeiro, Honrado \& Leal, 2004).

Foi realizada uma análise fatorial confirmatória (AFC) através do software AMOS. Recorreu-se ao método da máxima verosimilhança (Marôco, 2014). Para verificar o ajustamento do modelo foram utilizados os seguintes índices: rácio entre o Qui quadrado e os graus de liberdade (X2/g.l); goodness-of-fit index (GFI), root mean square error of approximation (RMSEA); comparative fit index (CFI) e Tucker-Lewis index (TLI) (Marôco, 2014; Sousa, Marques-Vieira, Carvalho, Veludo \& José, 2015).

Estes índices podem ser classificados em índices absolutos e índices relativos. Os índices absolutos avaliam a qualidade do modelo, sem comparar com outros modelos. Os índices mais utilizados nesta família (X2/g.l) são: quando for igual a 1 o ajustamento é perfeito, quando inferior a 2 o ajustamento é bom, é aceitável quando inferior a 5 e é inaceitável quando superior a 5. Root Mean Square Residual (RMSR) determina-se ao dividir a raiz quadrada da matriz dos erros, pelos graus de liberdade. Assim, quanto menor for o RMSR melhor será o ajustamento. Quando RMSR igual a 0 indica que o ajustamento é prefeito. O goodness of fit index (GFI) explica a proporção da covariância observada entre as variáveis manifestas, explicadas pelos modelos ajustados.
De uma forma geral, no que respeita ao GFI, considera-se que inferior a 0,8 indica modelos com mau ajustamento aos dados, entre 0,9 e 0,95 indica um bom ajustamento, superior a 0,95 indica ajustamento muito bom e igual a 1 ajustamento perfeito. Os índices relativos avaliam a qualidade do modelo sob teste, relativamente ao modelo com pior ajustamento possível e/ou ao modelo com o melhor ajustamento possível.

O comparative fit index (CFI) compara o ajustamento do modelo em estudo (X2) com os graus de liberdade (gl), com o ajustamento do modelo basal com os graus de liberdade. Geralmente aceita-se que para CFI inferior a 0,9 indica modelos com mau ajustamento, entre 0,9 e 0,95 indica um bom ajustamento, superior a 0,95 ajustamento muito bom e igual a 1 ajustamento perfeito. O Tucker-Lewis index (TLI), também conhecido por Bentler-Bonett-non-normed fit index (NNFI), em que os valores do TLI variam entre 0 e 1 . Os valores próximos de 1 indicam ajustamento muito bom (Marôco, 2014).

No respeito pelos princípios éticos foi concedida autorização para utilização pelos autores das versões portuguesas da DASS-21 (Ribeiro et al., 2004), bem como o estudo foi aprovado pela Comissão de Ética da Diaverum ( $\left.{ }^{\circ} 1 / 2015\right)$ e pela Comissão de Ética do Centro Hospitalar em causa (175/2015). Neste estudo os participantes foram informados sobre os direitos de garantia de sigilo dos seus dados, de desistência sem prejuízo para o próprio e assinarem o termo de consentimento livre e esclarecido.

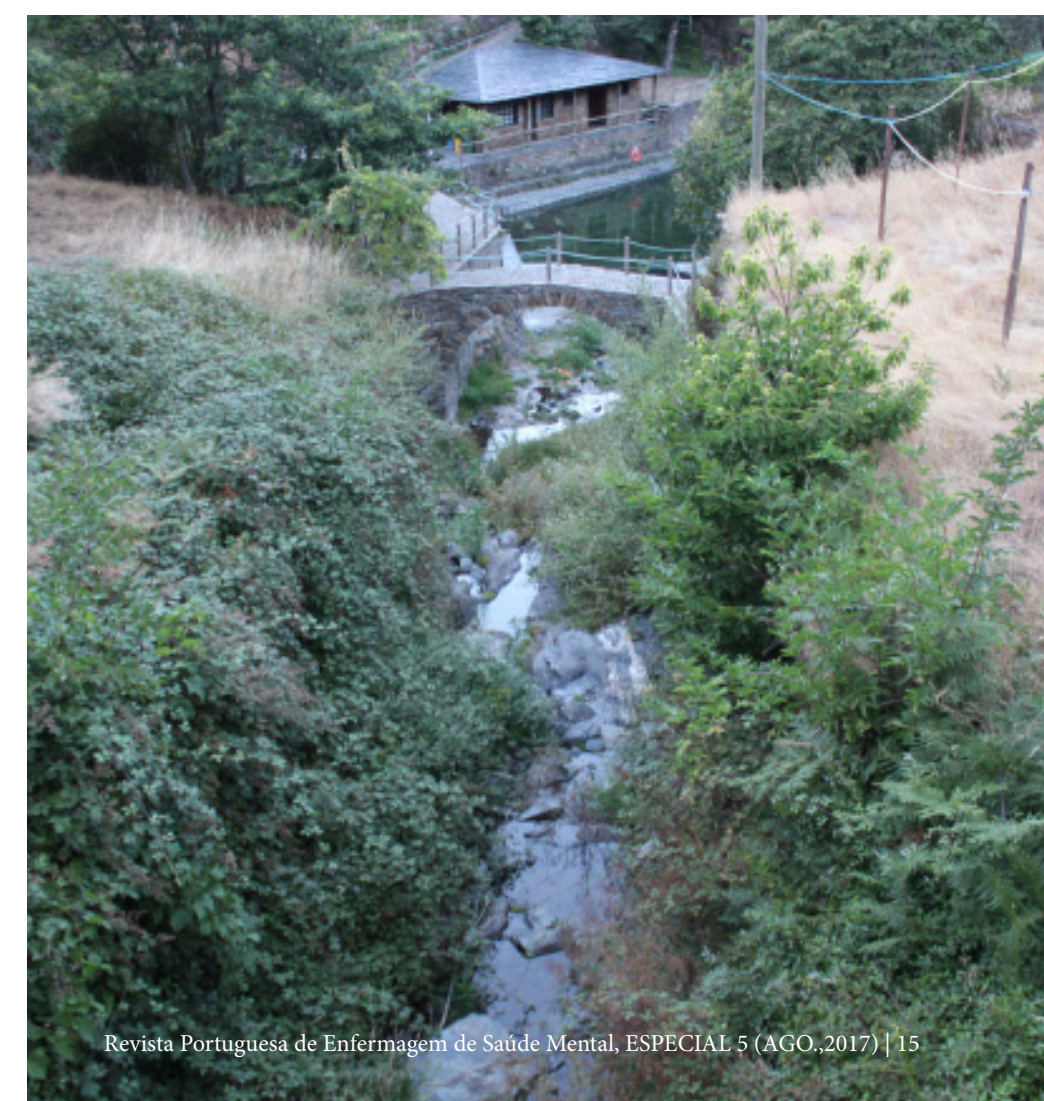




\section{RESULTADOS}

A amostra foi constituída por 159 pessoas que cumpriam os critérios de elegibilidade. O perfil sociodemográfico e de saúde encontra-se exposto na tabela 1.

Tabela 1 - Características das pessoas com DRC avaliadas nas duas clínicas

\begin{tabular}{|c|c|c|}
\hline Amostra & Frequência & Percentagem \% \\
\hline \multicolumn{3}{|c|}{ Sexo } \\
\hline Feminino & 62 & 62 \\
\hline Masculino & 97 & 97 \\
\hline \multicolumn{3}{|c|}{ Local de colheita de dados } \\
\hline Hospital & 12 & 7,5 \\
\hline Clínica 1 & 80 & 50,3 \\
\hline Clínica 2 & 67 & 42,2 \\
\hline \multicolumn{3}{|c|}{ Nacionalidade } \\
\hline Portuguesa & 127 & 80,4 \\
\hline Outra & 31 & 19,6 \\
\hline \multicolumn{3}{|c|}{ Escolaridade } \\
\hline Analfabeto & 3 & 1,9 \\
\hline $4^{\circ}$ Ano do Ensino Básico & 62 & 39,7 \\
\hline $6^{\circ}$ Ano de Escolaridade & 32 & 20,5 \\
\hline 9o Ano do Ensino Secundário & 23 & 14.7 \\
\hline 12 Ano do Ensino Secundário & 21 & 13,5 \\
\hline Licenciatura & 13 & 8,3 \\
\hline Mestrado e Doutoramento & 2 & 1,3 \\
\hline \multicolumn{3}{|c|}{ Atividade profissional } \\
\hline Reformado & 115 & 76,2 \\
\hline Ativo & 36 & 23,8 \\
\hline \multicolumn{3}{|c|}{ Estado civil } \\
\hline Solteiro(a) & 44 & 27,8 \\
\hline Casado(a) & 87 & 55,1 \\
\hline Viúvo(a) & 16 & 10,1 \\
\hline Divorciado/separado & 11 & 7,0 \\
\hline \multicolumn{3}{|c|}{ Hipertensão Arterial } \\
\hline Não & 61 & 38,9 \\
\hline Sim & 96 & 61,1 \\
\hline \multicolumn{3}{|c|}{ Diabetes mellitus } \\
\hline Não & 118 & 74,7 \\
\hline Sim & 40 & 25,3 \\
\hline
\end{tabular}

Nesta amostra, a média de idade era de 58,5 anos $( \pm 15)$. A maioria eram do sexo masculino $(61 \%)$, de nacionalidade portuguesa $(80,4 \%)$, tinham 4 anos de escolaridade $(39,7 \%)$, estavam reformados $(76,2 \%)$ e eram casados $(55,1 \%)$. Relativamente aos dados de saúde, estavam em programa de hemodiálise há 72,17 meses $( \pm 55,6), 61,1 \%$ apresentavam hipertensão arterial e $25,3 \%$ diabetes.

\section{Análise fatorial confirmatória}

Numa amostra de 159 pessoas, aplicou-se a AFC para a solução de dois fatores (figura 1). Fez-se uma covariação dos erros de algumas variáveis manifestas, estabelecendo entre si uma correlação moderada. Obteve-se X2/g.l=1,980, RMSEA=0,08, GFI=0,84, $\mathrm{CFI}=0,87 \mathrm{e}$ TLI $=0,85$.

Figura 1 - Modelo Bidimensional da DASS-21 em pessoas com DRC

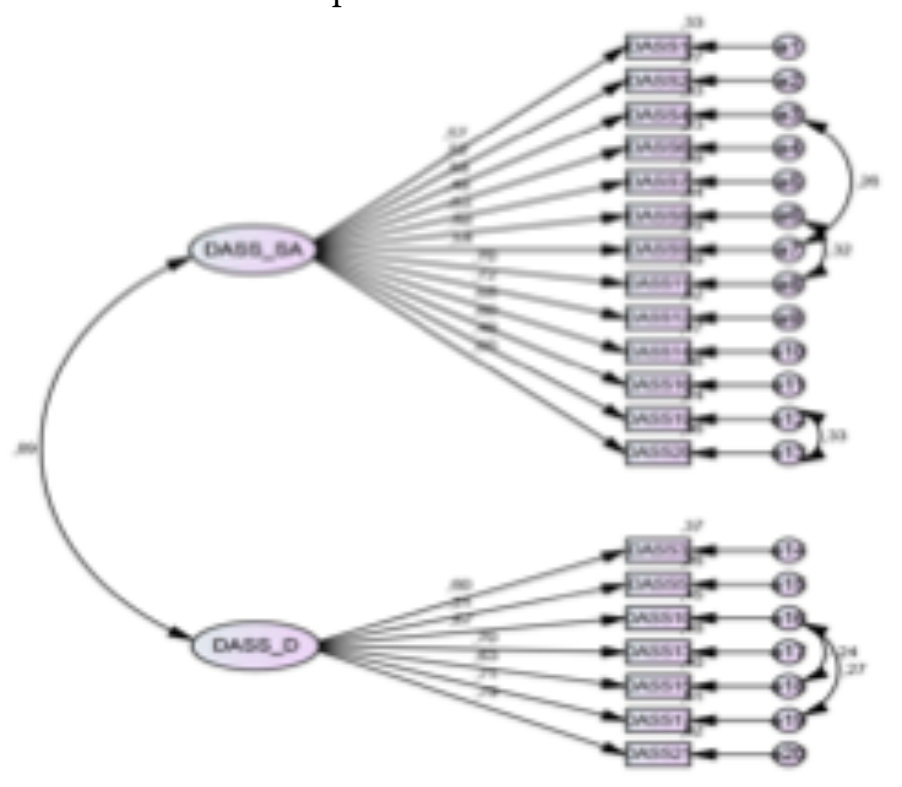

\section{DISCUSSÃO}

Os resultados da AFC demonstraram ter um bom ajustamento (Marôco, 2014) para a hipótese da solução de dois fatores, mas, os índices apresentam valores abaixo de 0,9 . Não se confirmou a solução proposta na versão original e na versão portuguesa (Ribeiro, Honrado \& Leal, 2004), mas confirmou-se uma estrutura idêntica à versão portuguesa do DASS$21 \mathrm{em}$ pessoas com doença mental (Apóstolo, Mendes \& Azeredo, 2006).

Neste estudo os resultados obtidos na AFC são diferentes dos encontrados nos estudos com versões da DASS-21 na língua inglesa (Sinclair et al., 2012) e em outras culturas (Oei et al., 2013; Tran et al., 2013) que apontam para uma estrutura de três fatores.

Estes resultados sugerem diferenças na estrutura quando comparadas com a versão portuguesa (Ribeiro, Honrado \& Leal, 2004) e com a escala original (Lovibond \& Lovibond, 1995), contudo aproxima-se de uma outra versão portuguesa que apresenta uma solução bifatorial (Apóstolo et al., 2006).

No que concerne às limitações deste estudo elas prendem-se, essencialmente, com a falta de financiamento e a dificuldade em conseguir uma amostra maior. 
Na realização desta investigação foi usada uma amostra representativa de pessoas com DRC em programa de hemodiálise. No entanto, recomenda-se em estudos futuros, a realização de uma AFC numa amostra superior a 300 pessoas.

\section{CONCLUSÃO}

A DASS-21, versão portuguesa, apresenta dois fatores, e é válida para medir stresse/ansiedade e depressão em pessoas com DRC.

\section{IMPLICAÇÕES PARA A PRÁTICA CLÍNICA}

Estas medidas de stresse/ansiedade e depressão podem ser integradas, no momento da avaliação inicial, na avaliação continua da monitorização e no final das intervenções de enfermagem para monitorizar ganhos sensíveis aos cuidados de enfermagem no âmbito da saúde e bem-estar.

\section{REFERÊNCIAS BIBLIOGRÁFICAS}

Apóstolo, J. L. A., Figueiredo, M. H., Mendes, A. C., \& Rodrigues, M. A. (2011). Depression, anxiety and stress in primary health care users. Revista Latino-Americana de Enfermagem, 19(2), 348-353. doi:10.1590/S010411692011000200017.

Apóstolo, J. L. A., Mendes, A. C., \& Azeredo, Z. A. (2006). Adaptation to Portuguese of the depression, anxiety and stress scales (DASS). Revista Latino-Americana de Enfermagem, 14(6), 863-871. doi:10.1590/ S0104-11692006000600006.

Bottesi, G., Ghisi, M., Altoè, G., Conforti, E., Melli, G., \& Sica, C. (2015). The Italian version of the Depression Anxiety Stress Scales-21: Factor structure and psychometric properties on community and clinical samples. Comprehensive psychiatry, 60, 170-181. doi: 10.1016/j. comppsych.2015.04.005.

Cavalcante, M. C. V., Lamy, Z. C., Santos, E. C., \& Costa, J. M. (2015). Portadores de doença renal crônica em fase produtiva: percepção sobre limitações resultantes do adoecimento. Revista de Medicina de Minas Gerais, 25(4): 484-492.
Costa, F. G., \& Coutinho, M. D. P. L. (2016). Síndrome depressiva: um estudo com pacientes e familiares no contexto da doença renal crônica. Estudos Interdisciplinares em Psicologia, 7(1), 38-55. doi:10.5433/22366407.2016v7n1p38.

Fassbinder, T. R. C., Winkelmann, E. R., Schneider, J., Wendland, J., \& Oliveira, O. B. D. (2015). Capacidade funcional e qualidade de vida de pacientes com doença renal crônica pré-dialítica e em hemodiáliseUm estudo transversal. Jornal Brasileiro de Nefrologia, 37(1), 47-54. doi: http://dx.doi.org/10.5935/01012800.20150008 .

Gomez, R., Summers, M., Summers, A., Wolf, A., \& Summers, J. J. (2014). Depression Anxiety Stress Scales-21: Factor structure and test-retest invariance, and temporal stability and uniqueness of latent factors in older adults. Journal of Psychopathology and Behavioral Assessment, 36(2), 308-317.

Lima, D. V. M. (2011). Research design: a contribution to the author. Online Brazilian Journal of Nursing, 10(2). Disponível em: http://www.objnursing.uff.br/index.php/nursing/article/view/3648/pdf_1.

Lovibond, P. F., \& Lovibond, S. H. (1995). The structure of negative emotional states: Comparison of the Depression Anxiety Stress Scales (DASS) with the Beck Depression and Anxiety Inventories. Behaviour Research and Therapy, 33(3), 335-343. doi:10.1016/00057967(94)00075-U.

Marôco, J. (2014). Análise de equações estruturais: Fundamentos teóricos, software \& aplicações. 2a Edição. Report Number, Lda.

Oei, T. P., Sawang, S., Goh, Y. W., \& Mukhtar, F. (2013). Using the depression anxiety stress scale 21 (DASS-21) across cultures. International Journal of Psychology, 48(6), 1018-1029. doi: 10.1080/00207594.2012.755535.

Pinho, N. A. D., Silva, G. V. D., \& Pierin, A. M. G. (2015). Prevalência e fatores associados à doença renal crônica em pacientes internados em um hospital universitário na cidade de São Paulo, SP, Brasil. Jornal Brasileiro de Nefrologia, 37(1), 91-97. doi: 10.5935/0101-2800.20150013. 
Pinto, J. C., Martins, P., Pinheiro, T. B., \& Oliveira, A. C. (2015). Ansiedade, depressão e stresse: um estudo com jovens adultos e adultos portugueses. Psicologia, Saúde \& Doenças, 16(2), 148-163.

Ribeiro, J. L. P., Honrado, A. A. J. D., \& Leal, I. P. (2004). Contribuição para o estudo da adaptação portuguesa das escalas de ansiedade, depressão e stress (EADS) de 21 itens de Lovibond e Lovibond. Psicologia, Saúde \& Doenças, 2, 229-239.

Santos, R. L. G., de Oliveira, D. R. F., Nunes, M. G. S., Barbosa, R. M. P., \& Gouveia, V. D. A. (2015). Evaluation of the knowledge of chronic renal patients in conservative treatment about dialytic modalities. Journal of Nursing UFPE on line, 9(2), 651-660. doi: 10.5205/ reuol.7028-60723-1-SM.0902201522.

Sinclair, S. J., Siefert, C. J., Slavin-Mulford, J. M., Stein, M. B., Renna, M., \& Blais, M. A. (2012). Psychometric evaluation and normative data for the depression, anxiety, and stress scales-21 (DASS21) in a nonclinical sample of US adults. Evaluation \& the Health Professions, 35(3), 259-279. doi: $10.1177 / 0163278711424282$.
Sousa, L. M. M., Marques-Vieira, C. M. A., Carvalho, M. L., Veludo, F., \& José, H. M. G. (2015). Fidelidade e validade na construção e adequação de instrumentos de medida. Enformação, 5, 25-32. Disponível em: http://www.acenfermeiros.pt/index. php?id $1=15 \&$ id $2=9$

Tran, T. D., Tran, T., \& Fisher, J. (2013). Validation of the depression anxiety stress scales (DASS) 21 as a screening instrument for depression and anxiety in a rural community-based cohort of northern Vietnamese women. BMC Psychiatry, 13(1), 24. doi: 10.1186/1471-244X-13-24.

Vignola, R. C. B., \& Tucci, A. M. (2014). Adaptation and validation of the depression, anxiety and stress scale (DASS) to Brazilian Portuguese. Journal of affective disorders, 155, 104-109. doi: 10.1016/j. jad.2013.10.031.

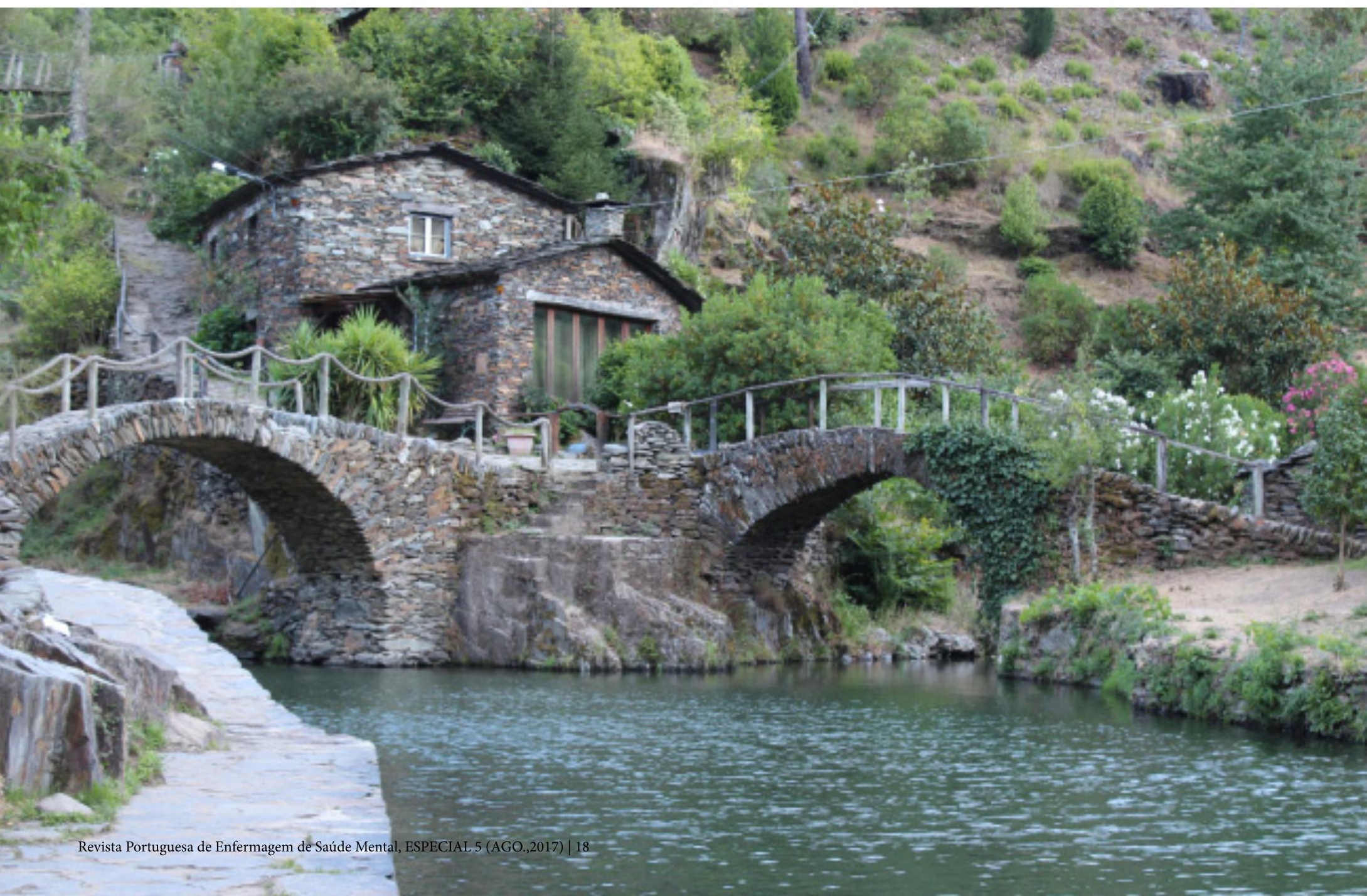

